OPEN ACCESS

Edited by: Alessio Merola,

Magna Græcia University, Italy

Reviewed by:

Qining Wang,

Peking University, China

Carmen C. Y. Poon,

The Chinese University of

Hong Kong, Hong Kong

*Correspondence:

M. Reza Motamedi mohammadreza.motamedi.1@ens. etsmtl.ca, reza.motamedi@robotmaster.com

Specialty section:

This article was submitted to

Biomedical Robotics,

a section of the journal

Frontiers in Robotics and $\mathrm{Al}$

Received: 20 November 2016 Accepted: 03 February 2017

Published: 22 February 2017

Citation:

Motamedi MR, Florant $D$ and Duchaine V (2017) Comparing the Exteroceptive Feedback of Normal Stress, Skin Stretch, and Vibrotactile Stimulation for Restitution of Static

Events.

Front. Robot. Al 4:6. doi: 10.3389/frobt.2017.00006

\section{Comparing the Exteroceptive Feedback of Normal Stress, Skin Stretch, and Vibrotactile Stimulation for Restitution of Static Events}

\author{
M. Reza Motamedi*, David Florant and Vincent Duchaine \\ Control and Robotics Laboratory (CoRo), Department of Automated Production Engineering, École de Technologie \\ Supérieure (ÉTS), Montreal, QC, Canada
}

This paper investigates the effectiveness of three types of haptic feedback: normal stress, tangential force, and vibrotactile stimulation. Modern prosthetic limbs currently available on the market do not provide a wide range of sensory information to amputees, forcing amputees to mainly rely on visual attention when manipulating objects. We aim to develop a haptic system that can convey information to the central nervous system (CNS) through haptic feedback. To this end, we aim to find out which type of feedback performs best under static conditions, so that it can be used to restore a sense of grasping force to amputees. We tested the three main stimulation methods by inputting a series of five force magnitudes to each haptic device, so that the device applied the corresponding feedback to the participants' finger pads. The participants then pressed on a force sensor, with the goal of applying the same level of force to a force sensor as they believed the haptic device had initially conveyed to them via their finger pads. While the subjects pressed on the force sensor, the haptic device applied a level of feedback to their forearms that corresponded to the pressure they were applying to the sensor. These tests provided fifteen numerical data per subject and a total of 180 trials for all twelve subjects. The end results indicate that even though all the stimulation methods provided a sufficient level of feedback, normal stress seems more effective than either tangential force or vibrotactile stimulation, at conveying the sense of pressure to the finger pad.

Keywords: mechanoreception, tactile sensitivity, static modalities, exteroception, haptics

\section{INTRODUCTION}

Prosthetic upper limbs require the user to establish an adequate safety margin during objectmanipulation tasks, in order to prevent object damage or slippage (Damian et al., 2012). For amputees, this safety margin is primarily determined by visual attention, as current commercially available prosthetic limbs are not equipped with full tactile sensing capabilities. Visual attention alone, however, leaves much to be desired when it comes to grasping force regulation. For instance, most amputees are unable to hold an egg for more than a few seconds. They either apply too much 
pressure and break the shell or apply too little pressure and have it slip from their grasp.

Healthy humans, by contrast, do not need to pay constant visual attention (Davoodi and Loeb, 2012) during such tasks because the tactile sensors (mechanoreceptors) in their skin allow them to easily regulate the grasping force level (Ferrington et al., 1977; Dargahi and Najarian, 2004). The central nervous system (CNS) transmits proprioceptive and exteroceptive information whenever one comes into contact with an external stimulus; over time, these experiences are used to inform one's reaction to present events (Hager-Ross et al., 1996; Augurelle et al., 2003). The regulation of grasping force thus depends on having adequately regulated this force during similar events in the past.

The challenge we face today is to overcome the lack of sensory perception in prosthetic limbs, especially upper ones, so that amputees can have the same object-manipulation capabilities as unimpaired people. One way to accomplish this is through haptic feedback (Okamura et al., 1998; Lee et al., 2004; Bark et al., 2009), which provides tactile information to the user through the sense of touch. A haptic feedback system consists of several components. First, the prosthetic fingers can be fitted with sensors to provide tactile information to amputees (Beebe et al., 1998; Tanaka et al., 2007). The sensors' signals are then converted to voltage levels and input to the haptic device, which applies feedback to a healthy area of the user's skin (e.g., back of the neck, upper arm). Haptic feedback thus allows the user to recognize the level of grasping force, or other type of information, that is applied by the prosthetic hand to the object (Kaczmarek et al., 1991; Caldwell et al., 1999).

Haptic technology is a perceptual system, and it typically involves active manual exploration mediated by cutaneous and kinesthetic afferent subsystems (Lederman and Klatzky, 1999). Their combined functioning generates information to the user by detecting properties such as an object's shape, temperature, or surface texture. Thus, identifying the functionalities of different stimuli, like normal and shear forces and vibration, may lead to developing a haptic device with better performance in various environmental modalities (Bolanowski et al., 1988).

Perhaps the first idea that comes to mind is that the best type of stimulation will be the one that is most similar to the conditions. In this view, static feedback will be most effective for conveying static events such as grasping force, whereas dynamic feedback will be best for conveying dynamic events such as texture recognition. However, this view has not been unequivocally supported in the literature.

To date, various approaches in the field of haptics have explored different ways of stimulating the cutaneous receptors (Edin and Johansson, 1995; Caldwell et al., 1999; Martinez et al., 2011). Regarding the type of stimuli, the relative effectiveness of tangential versus normal displacement was studied in Biggs and Srinivasan (2002). The authors believe tangential stimulation is the superior choice for hairy skin, whereas normal force is more effective for the finger pads when the actuator is limited primarily in terms of peak displacement. Another study (Bark et al., 2008) found that skin stretch is superior to vibrotactile stimuli when the experiment is conducted under low inertia and velocity. Other studies (Gould et al., 1979; Srinivasan et al., 1990) have concluded that the finger pad is better at sensing tangential displacement than any other type of displacement, and in parallel the perceived intensity of tangential stimuli is linearly correlated with increases in force magnitude (Paré et al., 2002). Following this, our review of the literature does not reveal much information about exteroceptive feedback, especially when applied to the upper limb, where the restitution of steady pressure under static conditions is the main concern.

In this paper, we seek to determine the most effective type of haptic feedback-whether normal stress, tangential force, or vibrotactile stimulation-for conveying a level of force that is applied to the participants' finger pads. Our main goal is to use haptic feedback to alert amputees about how their prosthetic limb is interacting with the environment. The results could pave the way for further research in developing a prosthetic upper limb system that uses haptic feedback to compensate for the lack of touch sensitivity.

To this end, we begin by describing the technological components of our experiment, specifically the actuator and vibration motor that we used. We then discuss the procedure of the experiment, including the participants and the exact method that was used to produce the static and dynamic stimuli. Next, we present our results and explore the functionality of each type under static conditions. Finally, we conclude with a discussion of the factors influencing our results and explore how future work might improve upon the current study.

\section{PARTICIPANTS AND METHODS}

As can be seen from Figure 1, the experiment was performed using three types of feedback: normal force, tangential force, and vibrotactile stimulation. Each type was applied proportionally to the glabrous skin of the human forearm. The protocol of our experiment was approved two times in 2012 and 2015 by the Ethics Committee of Research (Comité d'éthique de la recherche or CÉR) at École de Technologie Supérieure (ÉTS), Montréal, QC, Canada.

\subsection{Participants}

The subject population consists of six men and six women, ranging in age from 22 to 34 . The majority of the participants were recruited from among the students of ÉTS. They were informed of the procedures prior to starting the experiment, and all signed the participation form.

\subsection{Haptic Devices}

\subsubsection{Vibrotactile Apparatus}

The vibrotactile stimulator we used is an unbalanced linear vibrator called the Haptuator (Figure 2) made by Tactile Labs, Inc. (Yao and Hayward, 2010). The Haptuator is a vibrotactile transducer with a bandwidth of $50-500 \mathrm{~Hz}$, and it depends on frequency as well as voltage for the oscillation of mass in its formation. For better performance, a signal source was used to supply a sine/ square wave with various frequencies and amplitudes. Because this device has a low input impedance $(8 \Omega)$, an op-amp was used for impedance matching to avoid current loading and voltage fading. During the experiment, the signal was transmitted from 


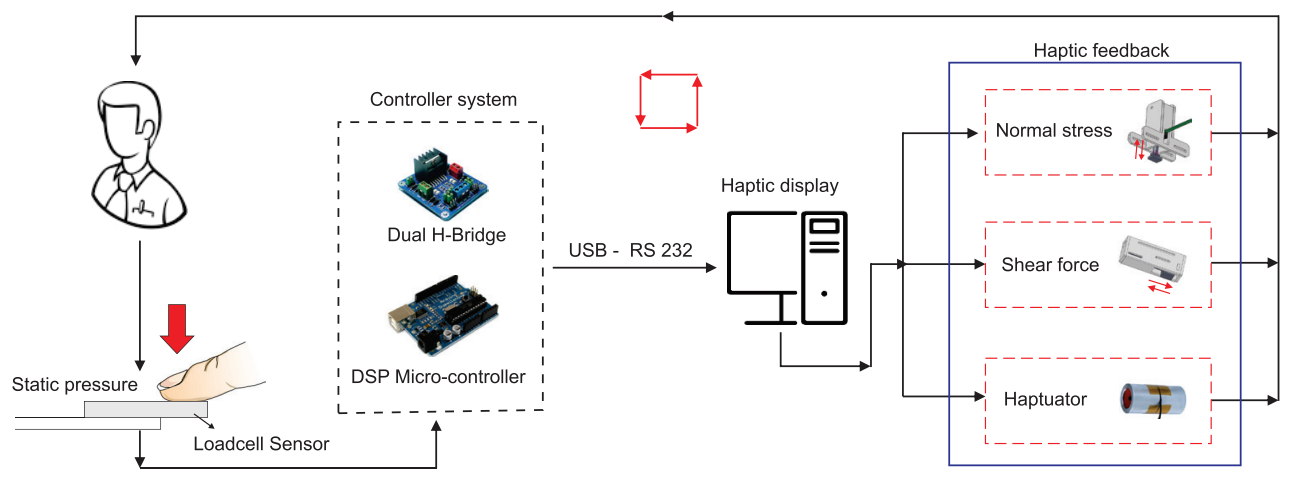

FIGURE 1 | Architecture of the experiment.

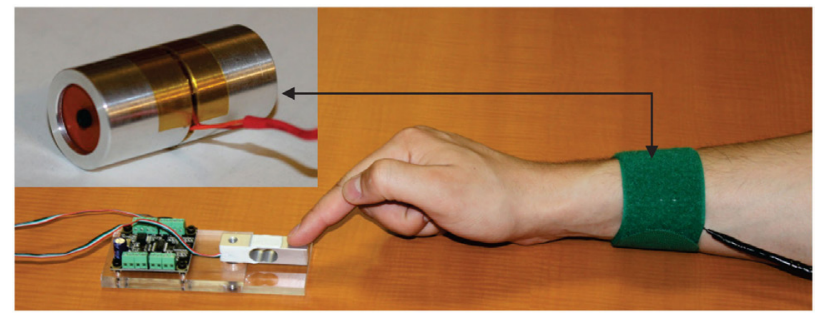

FIGURE 2 | Linear unbalanced vibration motor used to produce vibrotactile stimulation.

a function generator to a signal conditioner, the LM675 IC opamp, to provide vibration at the output. A serial communication cable (RS-232) was used to form a loop between the actuator, the microcontroller (DSP 56807), and the user interface.

\subsubsection{Normal and Tangential Force Apparatus}

Two miniature linear-motion actuators (PQ12 from Firgelli Technologies, Inc.) were used for this stage of the experiment. We used one of the actuators for the normal stress device and the other for the tangential force-application device. The PQ12 features position feedback to provide more sophisticated position control and is capable of pulling a load along its full stroke length. The input voltage of the actuator was $6 \mathrm{~V} \mathrm{DC}$, with maximum load of $20 \mathrm{~mm}$. This enabled us to determine the speed of movement of the end effector. When power is cut off, the actuator will hold its position, unless the load is applied in the back-drive. The final prototypes of the two devices are shown in Figures 3 and 4.

Figure 5 shows the specifications of the PQ12 actuator. The three gearing options $(30: 1,63: 1,100: 1)$ give us the possibility of having various force configuration ratios. Each force configuration ratio represents a trade-off between force and motion. The ratio of 30:1 is lower in force but faster in motion; ratio 63:1 provides moderate levels of both speed and motion; and 100:1 is higher in force but slower in motion.

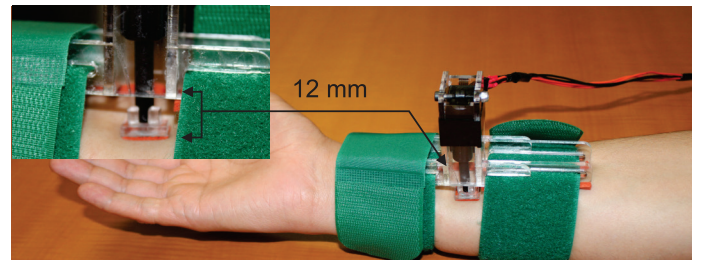

FIGURE 3 | Application of normal stress (vertical force) to the glabrous skin area.

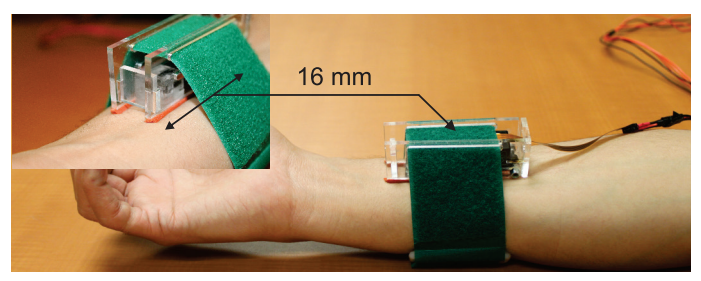

FIGURE 4 | Application of tangential force (shear) to the glabrous skin area.

\section{EXPERIMENT}

\subsection{Stimuli}

Participants were manually exposed to the three types of stimuli. In both normal stress and tangential force tests, forces were delivered at five different levels ranging from 2 to $10 \mathrm{~N}$ (increasing in increments of $2 \mathrm{~N})$. A rectangular unit $(14 \mathrm{~mm} \times 10 \mathrm{~mm})$ was placed at the head of the piston to act as an end effector. This was done for both the normal and the tangential devices in order to enlarge the area of contact between the piston and the participants' skin.

In the tangential force device, the initial position of the rectangular unit was set at $8 \mathrm{~mm}$ away from the actuator. This gap was left in order to avoid inducing pain by pinching the participants' skin. The maximum distance traveled by the piston was $12 \mathrm{~mm}$. 
Also, during the skin stretch tests, double-sided adhesive tape was used to attach the device to each participant's skin.

The vibration frequency for the vibrotactile test was set at $250 \mathrm{~Hz}$, which corresponds to the level of maximum human touch sensitivity (Bark et al., 2008; Wheeler et al., 2010). Most cell phones today are built to operate at this vibration threshold, because this is thought to maximize the chance of being noticed by the user while ringing in vibration mode. As shown in Figure 6, in our vibrotactile experiment five different input voltages $(2-10 \mathrm{~V})$ were delivered to the Haptuator. This allowed us to study a wide range of input voltages while observing how the feedback was received by the participants.

\subsection{Procedure}

At the start of the experiment, each participant was seated comfortably at a table. The participant's dominant hand was placed on an inflatable pillow, and the device was strapped to his or her forearm (Figure 7). Participants wore noise-canceling headphones and had their eyes covered with blindfolds to allow them to concentrate on their tasks without the influence of environmental events. To assist with force scaling, participants were familiarized with the various levels of the force stimuli during trial sessions.

During the experiments, the participants were asked to replicate the amount of feedback that each device applied to their forearms by pressing on a force sensor with their finger pads. The participants were not aware of the actual voltage levels that were input to each device and the order in which the devices were tested until all the experiments were complete.

We began the vibrotactile tests by running one of the input voltages (randomly selected from 2, 4, 6, 8, or $10 \mathrm{~V}$ ) into the Haptuator for about $15 \mathrm{~s}$. The Haptuator then applied vibrotactile
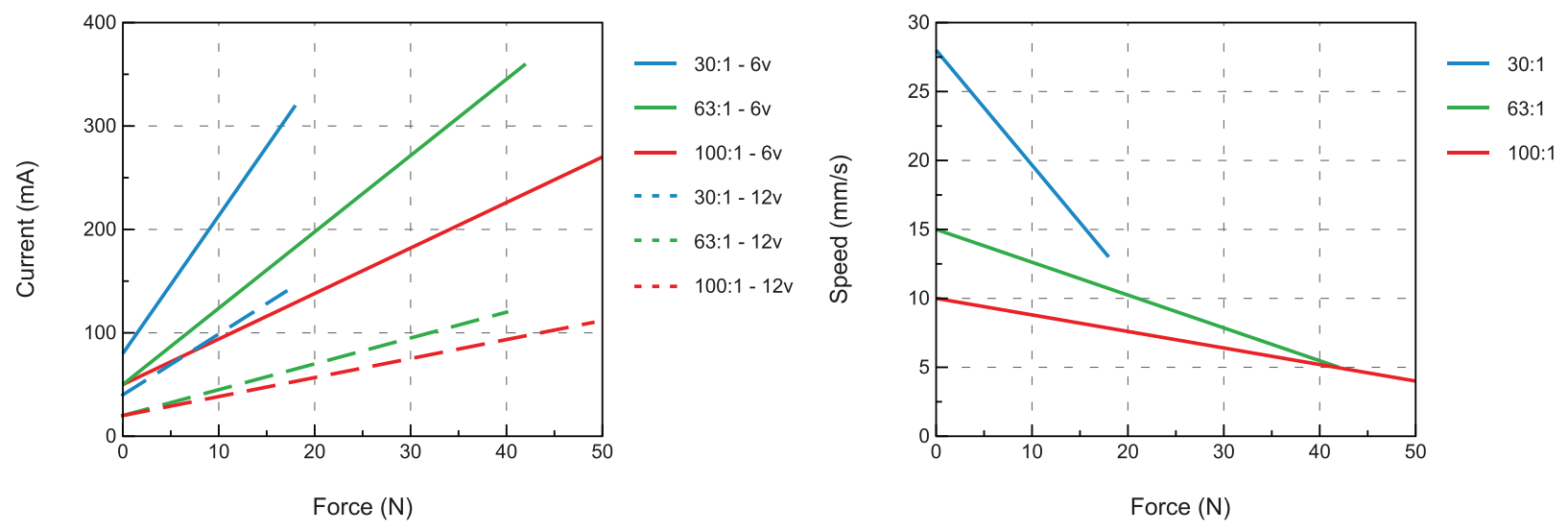

FIGURE 5 | Specifications of the PQ12 actuator under different gearing forces. Gear reduction ratio (refers to load curves above): 30,63 , and 100. Note that lower ratios are faster but provide less force and vice versa. 6,12 refer to the DC volts.

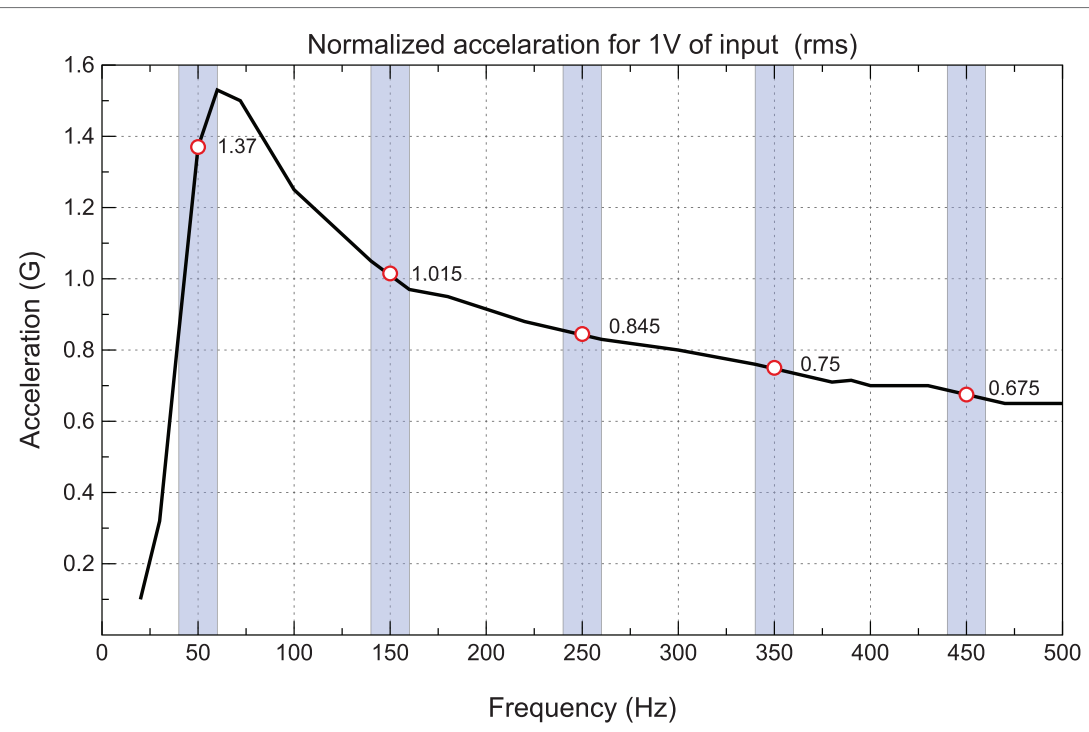

FIGURE 6 | Normalized acceleration of the Haptuator for $1 \mathrm{~V}$ of input. 


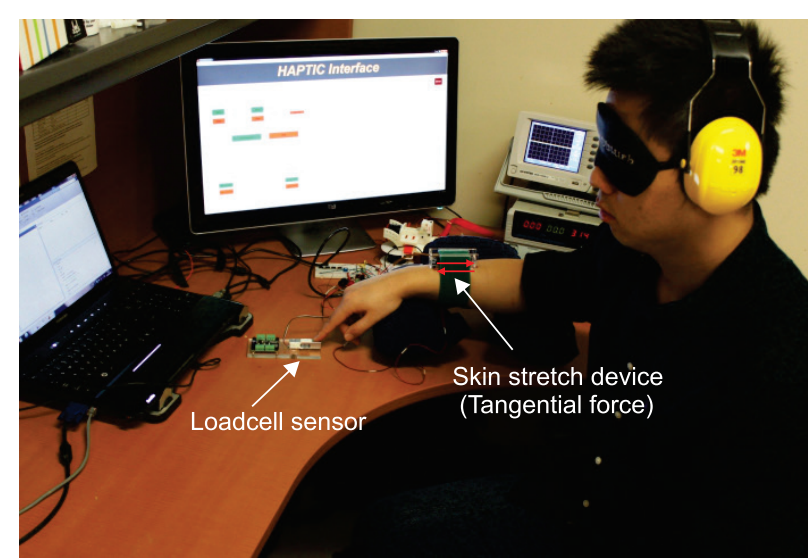

FIGURE 7 | Subject applying force to the load-cell sensor.

feedback, corresponding to this voltage level, to the participant's forearm. Immediately afterward, the participant was asked to press on the load-cell force sensor with his or her finger pad. The amount of force the participant applied to the load-cell force sensor was then transferred to the Haptuator, and the Haptuator again applied vibrotactile feedback, corresponding to this new voltage level, to the participant's forearm. The participant's task was to apply enough pressure to the force sensor so that the device would apply the same level of feedback as it did the first time.

The tests for the normal stress and tangential feedback devices proceeded in much the same way, except that the Haptuator was replaced by either a vertical force actuator for normal stress or a tangential force actuator for shear stress. As mentioned above, the order in which each device and voltage level was tested varied randomly for each participant. Participants were given a few moments to recover in between tests in order to prevent skin numbness. By the end of the experiments, each of the five magnitude levels was thus used as an input three times, providing fifteen numerical data per participant, and a total of 180 trials for all twelve participants.

When the participants' tasks were over, they were asked simple questions about the effectiveness of the feedback they received from each device. Finally, all the participants were presented with a graph showing the results of the experiment.

\section{RESULTS AND ANALYSIS}

\subsection{General Analysis}

Figure 8 displays diagrams, which represent the normal stress, tangential force, and the vibrotactile stimulation feedback from a participant, as a sample for each input level. The straight lines represent the desired level of the forces that were applied from the devices, and the margins around the lines indicate the feedback from the participants at that specific level. All collected data were statistically analyzed to determine the type of stimulation that best provides exteroceptive feedback under static conditions. Note that in order to avoid transient false data, only the last $10 \mathrm{~s}$ of each experiment were used in our statistics.

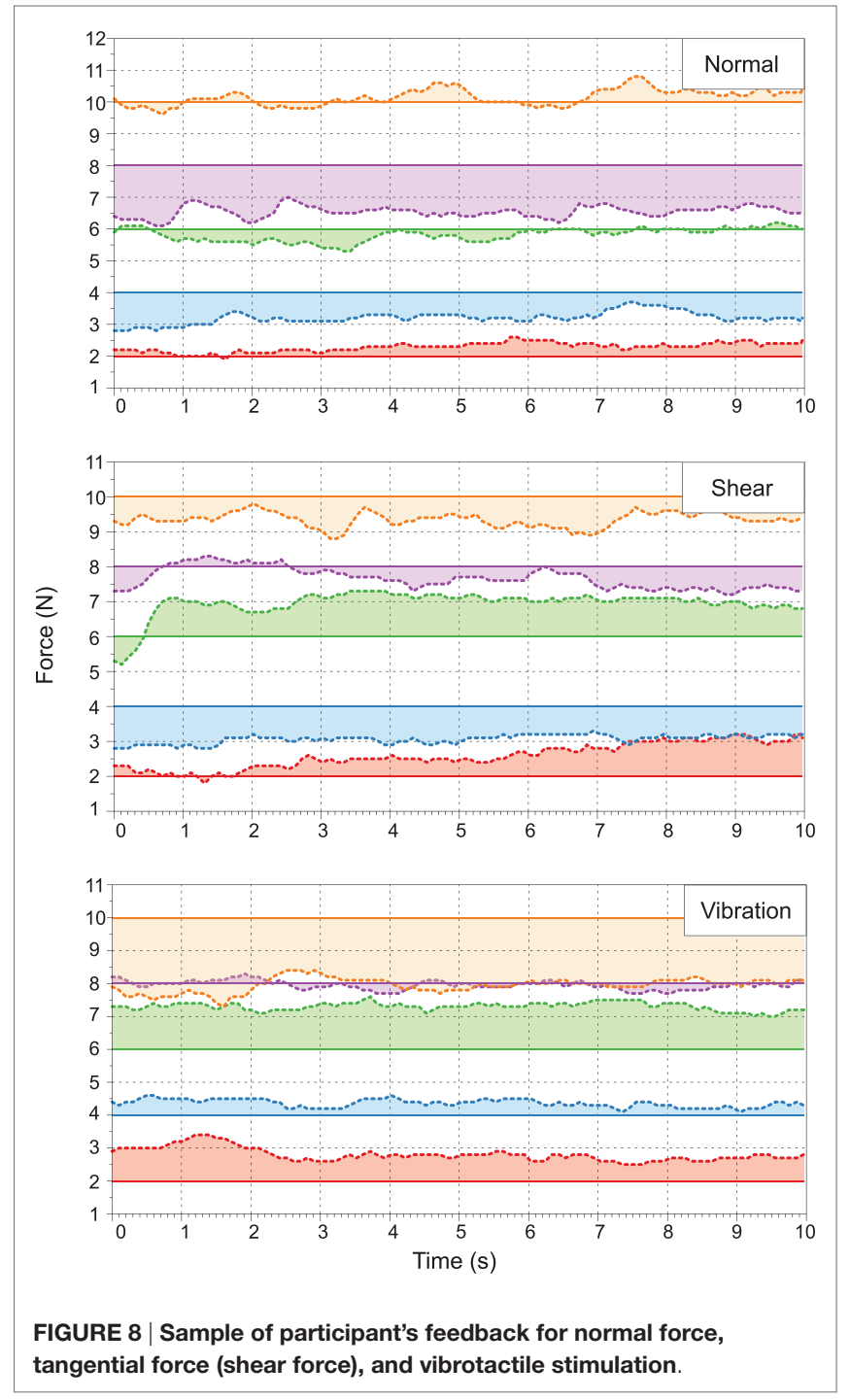

When we gathered the feedback from the twelve participants; it was clear that all the stimulation methods provide acceptable feedback. However, when we examined the data based on the critical statistical parameters, normal stress lead to a better restitution of static conditions than the other two types of stimulation at all five force levels. This can be observed from both Table 1 and Figure 9.

Out of the five input voltages, three $(2,4$, and $8 \mathrm{~V})$ have absolute means and variances that strongly show the slight superiority of normal stress. At these input levels, the second best type of feedback is vibrotactile, while tangential comes in last place. However, while normal stress is the clear winner, the errors for vibrotactile and tangential feedback are overall quite similar, and aside from perhaps at $6 \mathrm{~V}$, they likely do not differ significantly.

\subsection{Norm vs. Variance}

A critical parameter that should be considered in our analysis is the error based on variances and norms. As smaller variances 
and norms indicate smaller error in the feedback from the human participants, here we seek to find the best type of feedback by comparing the Manhattan, Euclidean, and Infinite norms for each type. Figure $\mathbf{1 0}$ shows the results of this comparison, in which each participant's individual feedback, along with the average feedback for each stimulus from all twelve participants, is distinguished by its variance versus the desired norms.

Again, the haptic device that applies normal stress appears to result in the smallest error, based on the average of norms and variances among all three types. This superiority remains the same in all types of calculated norms.

TABLE 1 | Statistical analysis of the collected data for the three types of stimulation.

\begin{tabular}{llccccc}
\hline Types & Statistics & $\mathbf{2}$ & $\mathbf{4}$ & $\mathbf{6}$ & $\mathbf{8}$ & $\mathbf{1 0}$ \\
\hline Normal force (N) & Mean & 2.32 & 3.44 & 6.39 & 7.43 & 9.81 \\
& SD & 0.54 & 0.41 & 0.65 & 0.42 & 0.68 \\
& Variance & 0.08 & 0.29 & 0.52 & 0.26 & 0.14 \\
Tangential force (N) & Mean & 2.88 & 4.96 & 6.54 & 6.99 & 10.7 \\
& SD & 0.39 & 0.65 & 0.63 & 0.44 & 0.13 \\
& Variance & 0.26 & 1.8 & 0.65 & 0.29 & 0.41 \\
Vibrotactile (N) & Mean & 2.62 & 4.68 & 7.14 & 7.18 & 9.17 \\
& SD & 0.14 & 0.14 & 0.05 & 0.34 & 0.49 \\
& Variance & 0.41 & 0.02 & 0.73 & 0.27 & 0.20
\end{tabular}

We also conducted an ANOVA test with a threshold of 95\% to determine whether the mean of the position error was significantly different at different levels (Table 2). The test statistics for our hypothesis (i.e., that normal stress is superior to vibrotactile and tangential force stimulation) do indeed show that there is a significant difference between the norms of the collected data $\left(\mathrm{F}=9.68, F_{0.055 .66}=2.35, \alpha=0.05\right.$, and $\mathrm{P}=$ 5.7E-07).

\subsection{Device Performance}

We also evaluated the best type of stimulation by comparing the performances of each participant during the tests. These results are shown in Figure 11. As was anticipated, normal stress is the most functional, with six participants having their best performance with this type, five in the middle, and only one performing worst. Vibrotactile stimulation is in second place, with four subjects performing best, three in the middle, and five performing worst. Finally, tangential force feedback is evidently the least effective of the three, since only two participants performed best while using it, four were in the middle, and six performed worst.

\subsection{Participants' Success Rates}

As mentioned earlier, at the end of each participant's tasks we questioned them about how they felt during the experiments. We
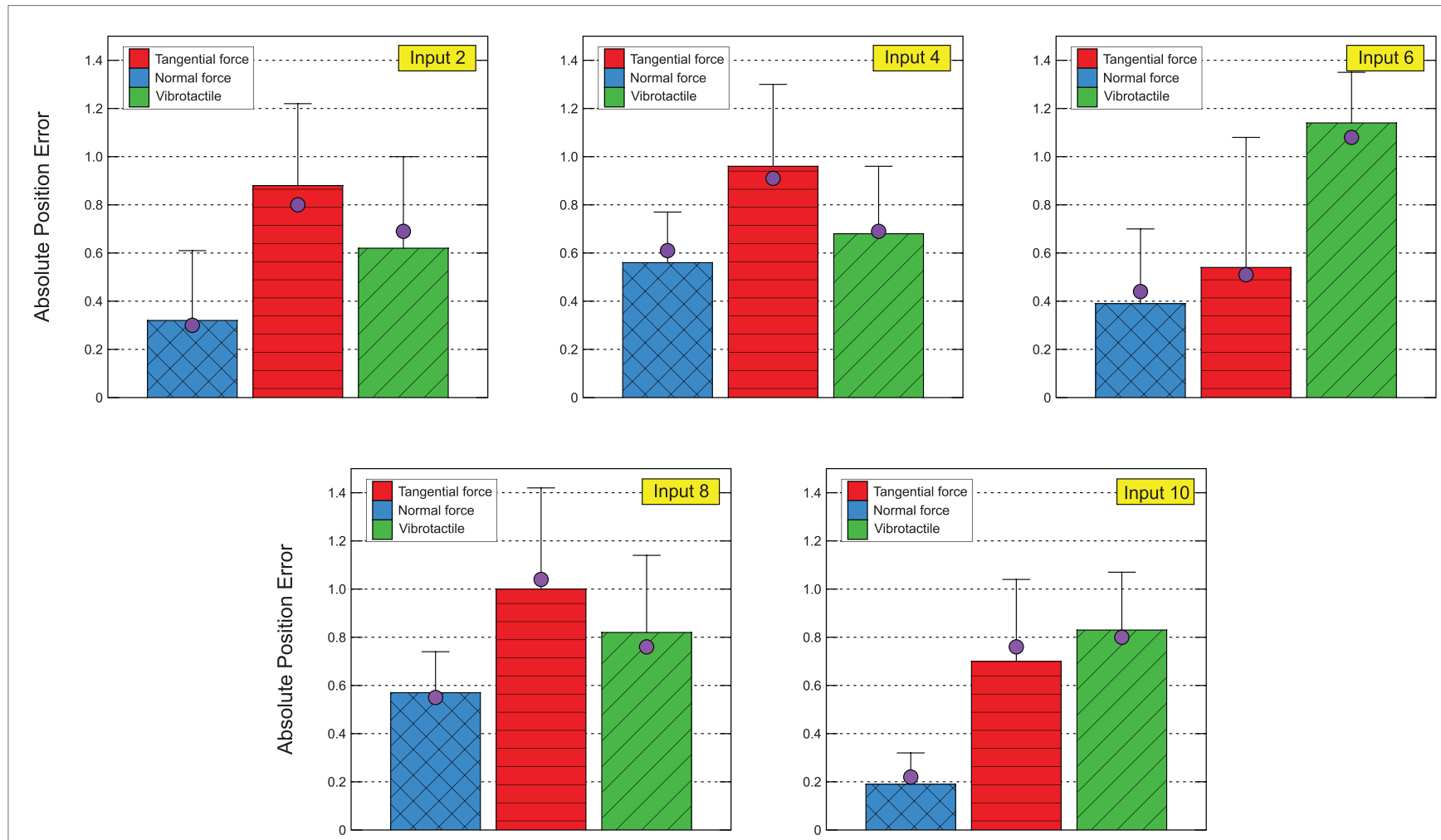

FIGURE 9 | Participants' feedback under the three types of stimulation. The bars show the absolute mean, the lines show the absolute error, and the purple circles indicate the median for all tests. 

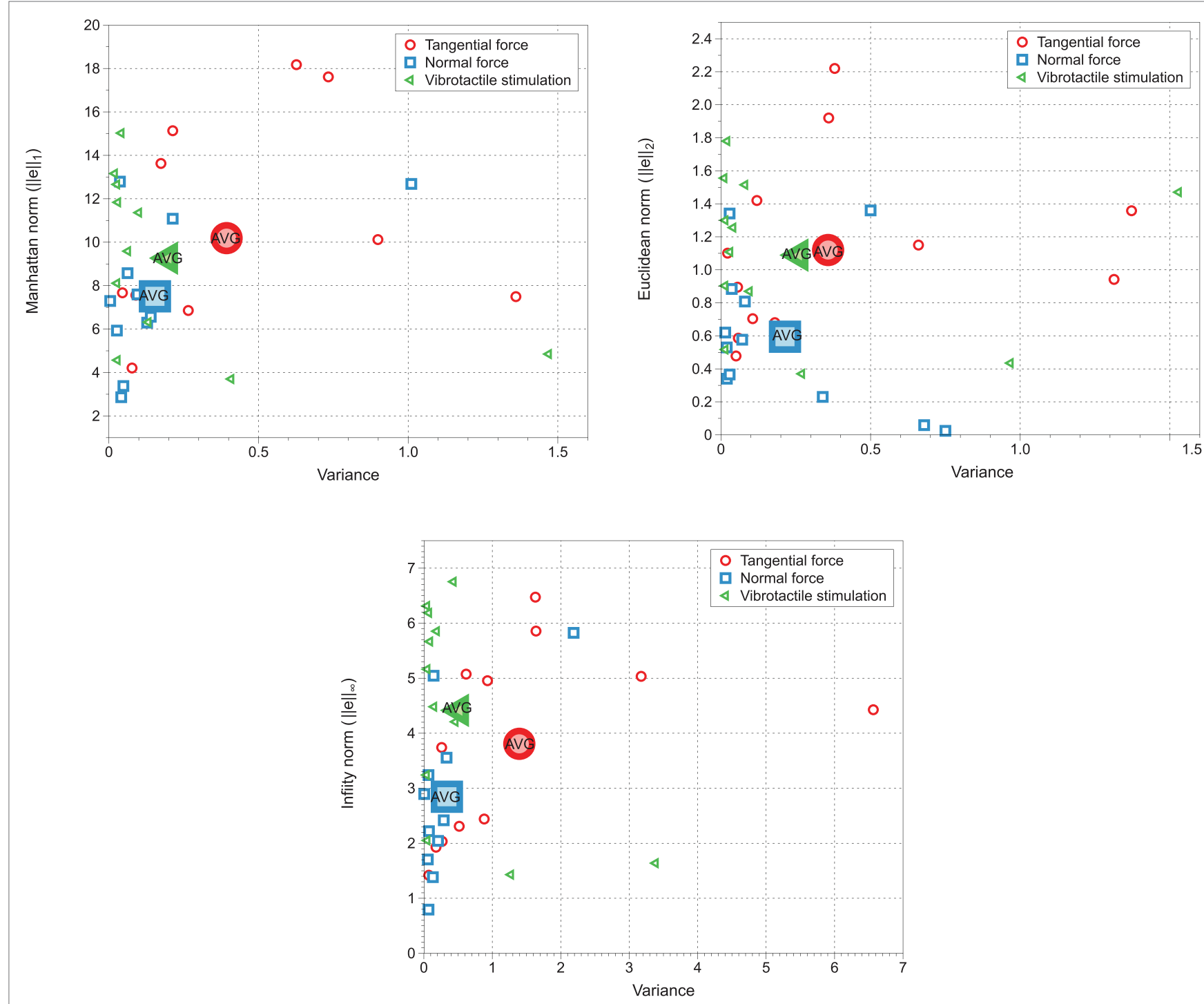

FIGURE 10 | Manhattan norm analysis for the feedback provided by normal stress, skin stretch, and vibrotactile stimulation.

TABLE 2 | ANOVA table showing the significance of the differences between the participants' feedback for each type of stimulation.

\begin{tabular}{lcccccc}
\hline Types & SS & df & MS & F & $\boldsymbol{P}$-value & $\boldsymbol{F}_{\text {critical }}$ \\
\hline Between-groups & 12.63 & 5 & 2.52 & 9.68 & $5.7 \mathrm{E}-07$ & 2.35 \\
Within-groups & 17.21 & 66 & 0.26 & & & \\
\hline
\end{tabular}

asked them to rate each device's overall effectiveness on a scale of 0 to 10 based on their own experience and preferences. As shown in Figure 12, the participants' ratings are linked to their performances. Eight of them believed normal stress was the most suitable for restitution of steady pressure under static conditions. Three of them were not completely satisfied with normal stress, and just one subject did not like the feeling of normal stress at all. Clearly, participants preferred the type of feedback that was easiest for them to use.

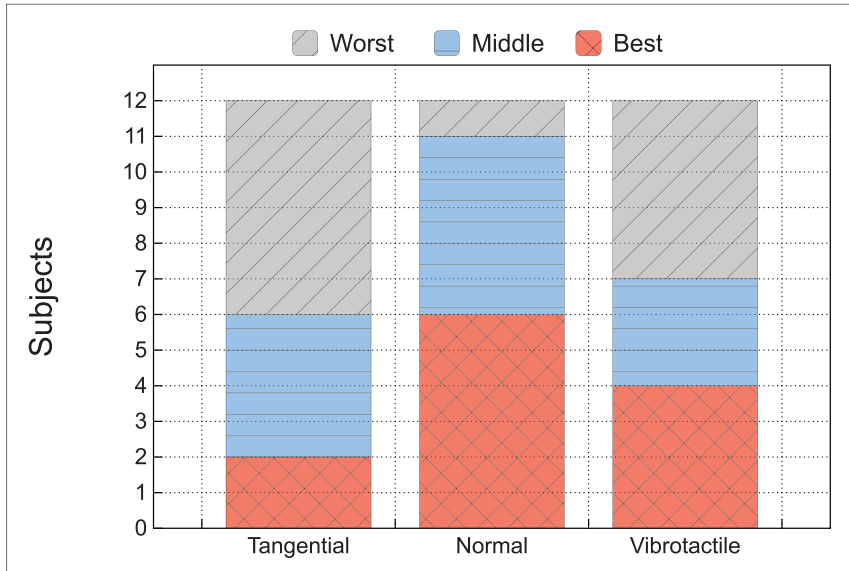

FIGURE 11 | Participants' performances with normal stress, skin stretch, and vibrotactile stimulation. 


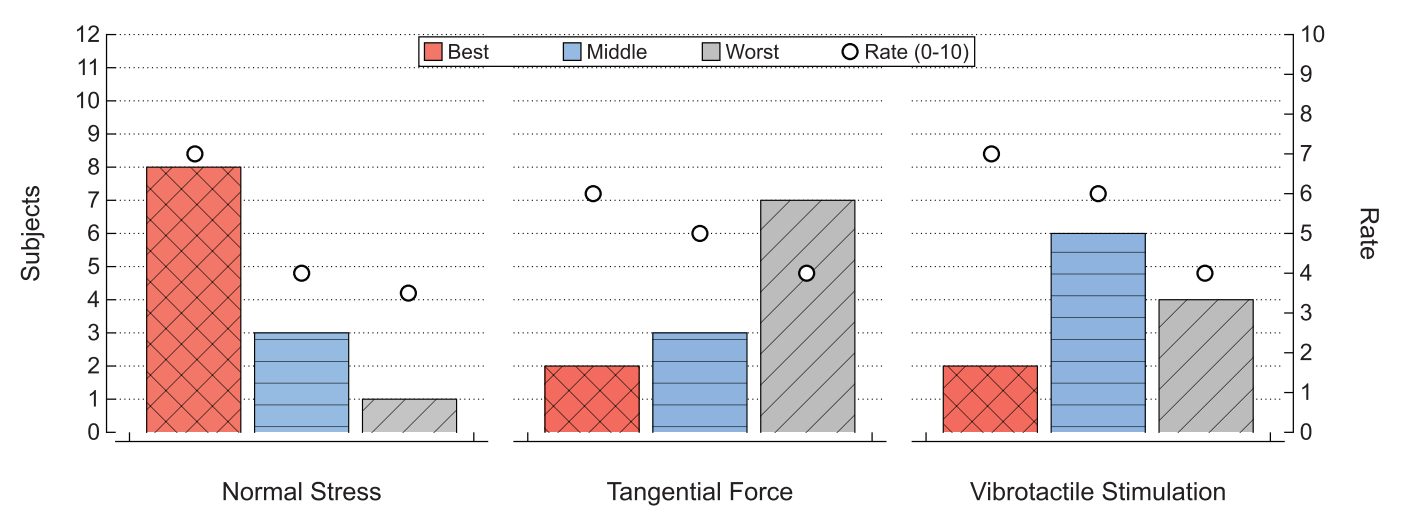

FIGURE 12 | Preference rating of different feedback conditions from the twelve participants.

During the rating process, several complaints came up that were repeated by many of the participants. Although they were familiar with vibrotactile stimulation and found it effective, they advised that it should not be applied for more than $5 \mathrm{~s}$. They generally agreed that vibrotactile stimulation is better for use in short-term conditions, such as conveying the sense of brief contact with an object, since they stated that the sensation quickly becomes annoying. The participants were more uncomfortable during the tangential force tests. Although we made sure that the tangential force device would not hurt the participants during the test, they reported feeling uneasy about the device because they feared it would stretch their skin too far. For this reason, we believe tangential force is the least suitable feedback method for our purposes. The normal stress stimulation, aside from being more effective overall, prompted the fewest complaints from participants. They liked that the device moved softly and smoothly over their skin, and that it conveyed the sense of pressure in a way that was easy to understand.

\section{CONCLUSION}

Human touch sensitivity is a complex biological system that deals with various environmental impacts. It is essential to use the proper stimulus for restoring touch sensitivity for a specific type of modality, like grasping. Obviously, the best answer may lie outside what can be found through simply comparing three different types of feedback; but we hope this study has been a step in the right direction.

Three of the most widely used types of haptic feedback were involved in our experiment. Although normal stress stimulation gave the best and most reliable feedback for restituting the static pressure applied to the finger pads, the other two, tangential force and vibrotactile stimulation, also provided acceptable results. When we look at the overall results and the participants' success rates, we see that many parameters affected their performances.
In vibrotactile stimulation, as mentioned earlier, the operation time plays a critical role. People are more interested in dynamic feedback for the purpose of being quickly alerted to some external event, and they prefer the vibration motor to not be in direct contact with their skin. We believe the participants were not as accurate when using vibrotactile feedback because they were not used to having a vibrator motor placed directly on their skin. When a cell phone vibrates, for instance, it is generally in a pocket or bag.

We found that it was relatively difficult to stimulate the tactile receptors via tangential force. The device required more precision, since a high level of pressure can be easily become annoying for the users. This became clear when we asked participants about their experience with the devices: many of them stated that the tangential device applied an uncomfortably high (though not painful) amount of pressure. Also, our experiment used tangential force applied to glabrous areas of the body; past researchers have found that it is more effective when applied to non-glabrous (i.e., hairy) skin (Biggs and Srinivasan, 2002).

Finally, normal stress seems highly functional and easy to recognize. This is likely due to its similarity to the pressing task and particularly to the applications we used in our experiment, Although normal stress comes with many advantages, we should take into consideration that applying too much pressure, such as more than $10 \mathrm{~mm}$ of skin displacement, can be as uncomfortable for the participants as skin stretch. In general, based on the participants' feedback, normal stress is highly recommended for conveying pressure of less than $10 \mathrm{~N}$. Within this limit, we can easily convey each $1 \mathrm{~N}$ increment of force by matching it to each millimeter of the distance traveled by the piston.

Taking everything into consideration, in order to design a haptic interface that can work under both dynamic and static conditions, the use of both normal stress and vibrotactile stimulation seems mandatory. Following their successful integration, the system can then be made yet more functional by adding 
tangential force and temperature sensors, in order to deal with dynamic as well as static modalities.

\section{AUTHOR CONTRIBUTIONS}

$\mathrm{MM}$ is the first author, and this is part of his $\mathrm{PhD}$ research entitled, "The use of multi-modal haptic feedback to convey various aspects of touch sensitivity." DF is the second author, and this is part of his MSc research. DF also involved in designing the

\section{REFERENCES}

Augurelle, A.-S., Smith, A. M., Lejeune, T., and Thonnard, J.-L. (2003). Importance of cutaneous feedback in maintaining a secure grip during manipulation of hand-held objects. J. Neurophysiol. 89, 665-671. doi:10.1152/ jn.00249.2002

Bark, K., Wheeler, J., Lee, G., Savall, J., and Cutkosky, M. (2009). "A wearable skin stretch device for haptic feedback," in World Haptics 2009 - Third Joint EuroHaptics Conference and Symposium on Haptic Interfaces for Virtual Environment and Teleoperator Systems (Reno: IEEE), 464-469.

Bark, K., Wheeler, J. W., Premakumar, S., and Cutkosky, M. R. (2008). “Comparison of skin stretch and vibrotactile stimulation for feedback of proprioceptive information," in 2008 Symposium on Haptic Interfaces for Virtual Environment and Teleoperator Systems (Reno: IEEE), 71-78.

Beebe, D., Denton, D. D., Radwin, R., and Webster, J. (1998). A silicon-based tactile sensor for finger-mounted applications. IEEE Trans. Biomed. Eng. 45, 151-159. doi:10.1109/10.661263

Biggs, J., and Srinivasan, M. (2002). "Tangential versus normal displacements of skin: relative effectiveness for producing tactile sensations," in Proceedings 10th Symposium on Haptic Interfaces for Virtual Environment and Teleoperator Systems. HAPTICS 2002 (Orlando, FL: IEEE), 121-128. doi:10.1109/ HAPTIC.2002.998949

Bolanowski, S. J. Jr., Gescheider, G. A., Verrillo, R. T., and Checkosky, C. M. (1988). Four channels mediate the mechanical aspects of touch. J. Acoust. Soc. Am. 84, 1680 .

Caldwell, D. G., Tsagarakis, N., and Giesler, C. (1999). "An integrated tactile/shear feedback array for stimulation of finger mechanoreceptor," in Proceedings 1999 IEEE International Conference on Robotics and Automation, Vol. 1 (Detroid: IEEE), 287-292.

Damian, D., Ludersdorfer, M., Kim, Y., Hernandez Arieta, A., Pfeifer, R., and Okamura, A. (2012). "Wearable haptic device for cutaneous force and slip speed display," in 2012 IEEE International Conference on Robotics and Automation (ICRA) (Saint Paul), 1038-1043.

Dargahi, J., and Najarian, S. (2004). Human tactile perception as a standard for artificial tactile sensing? A review. Int. J. Med. Robot. 1, 23-35.

Davoodi, R., and Loeb, G. (2012). Development of a physics-based target shooting game to train amputee users of multijoint upper limb prostheses. Presence 21, 85-95. doi:10.1162/PRES_a_00091

Edin, B. B., and Johansson, N. (1995). Skin strain patterns provide kinaesthetic information to the human central nervous system. J. Physiol. 487(Pt 1), 243-251.

Ferrington, D., Nail, B., and Rowe, M. (1977). Human tactile detection thresholds: modification by inputs from specific tactile receptor classes. J. Physiol. 272, 415-433.

Gould, W. R., Vierck, C. J. Jr., and Luck, M. M. (1979). "Cues supporting recognition of the orientation or direction of movement of tactile stimuli," in Sensory Functions of the Skin of Humans (New York: Springer), 63-78. mechanical devices used in this research and assisted through the experimental procedure. VD is the supervisor.

\section{FUNDING}

This work was supported by the Natural Sciences and Engineering Research Council of Canada (NSERC) and the Ministére du Développement économique, de l'Innovation et de l'Exportation du Québec (MDEIE).

Hager-Ross, C., Cole, K., and Johansson, R. (1996). Grip-force responses to unanticipated object loading: load direction reveals body- and gravity-referenced intrinsic task variables. Exp. Brain Res. 110, 142-150. doi:10.1007/BF00241383

Kaczmarek, K., Webster, J., Bach-y Rita, P., and Tompkins, W. J. (1991). Electrotactile and vibrotactile displays for sensory substitution systems. IEEE Trans. Biomed. Eng. 38, 1-16. doi:10.1109/10.68204

Lederman, S. J., and Klatzky, R. L. (1999). Sensing and displaying spatially distributed fingertip forces in haptic interfaces for teleoperator and virtual environment systems. Presence 8, 86-103.

Lee, J. C., Dietz, P. H., Leigh, D., Yerazunis, W. S., and Hudson, S. E. (2004). “Haptic pen: a tactile feedback stylus for touch screens," in Proceedings of the 17th Annual ACM Symposium on User Interface Software and Technology, UIST '04 (New York, NY: ACM), 291-294.

Martinez, J., Martinez, D., Molina, J., Gonzalez, P., and Garcia, A. (2011). "Comparison of force and vibrotactile feedback with direct stimulation for texture recognition," in International Conference on Cyberworlds (CW), 2011 (Banff), 62-68.

Okamura, A. M., Dennerlein, J. T., and Howe, R. D. (1998). "Vibration feedback models for virtual environments," in 1998 IEEE International Conference on Robotics and Automation, 1998 Proceedings, Vol. 1 (Leuven: IEEE), 674-679.

Paré, M., Carnahan, H., and Smith, A. M. (2002). Magnitude estimation of tangential force applied to the fingerpad. Exp. Brain Res. 142, 342-348. doi:10.1007/ s00221-001-0939-y

Srinivasan, M. A., Whitehouse, J., and LaMotte, R. H. (1990). Tactile detection of slip: surface microgeometry and peripheral neural codes. J. Neurophysiol. 63, 1323-1332.

Tanaka, Y., Sato, H., and Fujimoto, H. (2007). "Development of a finger-mounted tactile sensor for surface irregularity detection," in IEEE/RSJ International Conference on Intelligent Robots and Systems, 2007. IROS 2007 (San Diego), 690-696.

Wheeler, J., Bark, K., Savall, J., and Cutkosky, M. (2010). Investigation of rotational skin stretch for proprioceptive feedback with application to myoelectric systems. IEEE Trans. Neural. Syst. Rehabil. Eng. 18, 58-66. doi:10.1109/TNSRE. 2009.2039602

Yao, H.-Y., and Hayward, V. (2010). Design and analysis of a recoil-type vibrotactile transducer. J. Acoust. Soc. Am. 128, 619-627. doi:10.1121/1.3458852

Conflict of Interest Statement: The authors declare that the research was conducted in the absence of any commercial or financial relationships that could be construed as a potential conflict of interest.

Copyright (C) 2017 Motamedi, Florant and Duchaine. This is an open-access article distributed under the terms of the Creative Commons Attribution License (CC BY). The use, distribution or reproduction in other forums is permitted, provided the original author(s) or licensor are credited and that the original publication in this journal is cited, in accordance with accepted academic practice. No use, distribution or reproduction is permitted which does not comply with these terms. 